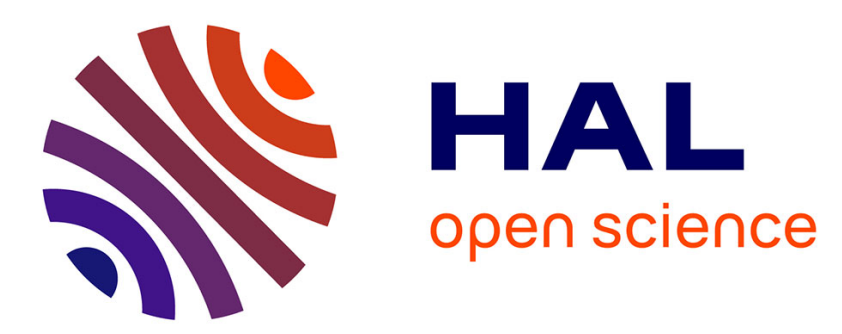

\title{
Measuring the order parameter of vertically aligned nanorod assemblies
}

\author{
Jeongmo Kim, Khalid Lahlil, Thierry Gacoin, Jongwook Kim
}

\section{To cite this version:}

Jeongmo Kim, Khalid Lahlil, Thierry Gacoin, Jongwook Kim. Measuring the order parameter of vertically aligned nanorod assemblies. Nanoscale, 2021, 13 (16), pp.7630-7637. 10.1039/d0nr08452b . hal-03334800

\section{HAL Id: hal-03334800 \\ https://hal.science/hal-03334800}

Submitted on 5 Sep 2021

HAL is a multi-disciplinary open access archive for the deposit and dissemination of scientific research documents, whether they are published or not. The documents may come from teaching and research institutions in France or abroad, or from public or private research centers.
L'archive ouverte pluridisciplinaire HAL, est destinée au dépôt et à la diffusion de documents scientifiques de niveau recherche, publiés ou non, émanant des établissements d'enseignement et de recherche français ou étrangers, des laboratoires publics ou privés. 


\section{ARTICLE}

\section{Measuring the Order Parameter of Vertically Aligned Nanorods Assembly}

Received 00th January 20xx, Accepted 00th January 20xx DOI: $10.1039 / x 0 x \times 00000 x$

\begin{abstract}
Jeongmo Kim, ${ }^{\text {a }}$ Khalid Lahlil, ${ }^{\mathrm{a}}$ Thierry Gacoin, ${ }^{\mathrm{a}}$ and Jongwook Kim ${ }^{* \mathrm{a}}$
Vertically aligned nanorods assemblies are of great interest both for fundamental studies of anisotropic physical properties arising from the structures and for the development of functional devices utilizing such anisotropic characteristics. Simultaneous measurement of the homeotropic order parameter $\left(S_{\text {homeo }}\right)$ of assemblies in dynamic states can allow further optimization of the assembly process and the device performance. Although many techniques (e.g. birefringence measurement, SAXS analysis, high-resolution microscopy) have been proposed to characterise $S_{\text {homeo, these do not yet }}$ meet the essential criteria such as for rapid, in situ and non-destructive analyses. Here, we propose a novel approach employing a unique photoluminescence behaviour of lanthanide-doped crystalline nanorods, of which the emission spectrum contains the detailed information on the structure of assembly. We demonstrate a rapid in-situ determination of $\mathrm{S}_{\text {homeo }}$ of $\mathrm{Eu}^{3+}$-doped $\mathrm{NaYF}_{4}$ nanorods of which the orientation is controlled under external electric field. The method does not require a consideration of polarization and can be performed using a conventional fluorescence microscopy setup. This new methodology would provide a more in-depth examination of various assembled nanostructures and the collective dynamics of their building blocks.
\end{abstract}

drying $^{14,15,16,17}$, shear stress under flow ${ }^{2,8,18,19}$, and externally applied electric en $11,13,20,21,22$ or magnetic fields $23,24,25$. Combination of these approaches has been useful to prepare active devices modulating strong anisotropic properties by reversibly controlling the director of organized particles with external fields (e.g. electro-optical device ${ }^{11,13,22}$ ). attracted much attention because of their unique anisotropic material properties such as optical retardation ${ }^{1,2}$, ferroelectricity and -magnetism ${ }^{3,4,5,6}$, polarized -luminescence and plasmons ${ }^{7,8,9,10}$. These properties are promising for their practical use in many functional devices. However, it has been a difficult problem to investigate and manipulate such properties because of the limited signal-to-noise ratio for individual nanorods and their random orientation in ensemble. Achieving significant intensities of response signals while getting access to the anisotropic properties inherent in individual particles becomes possible when considering aligned particles by self-assembly or directed assembly. Self-assembly can be achieved by controlling the sophisticated surface chemistry of colloidal nanorods, which often imparts an ability to be liquid crystalline. ${ }^{8,11,12,13}$ Directed assemblies are made by exerting forces, for instance, capillary forces during solvent

\footnotetext{
a. Laboratoire de Physique de la Matière Condensée, CNRS, École Polytechnique, Institut Polytechnique de Paris, 91128 Palaiseau, France
}

Corresponding Author:

thierry.gacoin@polytechnique.edu / jong-wook.kim@polytechnique.edu

Electronic Supplementary Information (ESI) available:

In-plane alignment of nanorods by electro-optical switching, Line shape comparison of $\mathrm{I}_{\pi}+\mathrm{I}_{\sigma}$ and $\mathrm{I}_{\pi+\sigma}$, Photoluminescence signal of $\mathrm{NaYF}_{4}$ :Eu nanorods as a function of relaxation time after the E-field turned off from the saturating E-field. Simulated profile of homeotropic order parameter analysis. $\mathrm{NaYF}_{4}: \mathrm{Eu}$ nanorods characterization. See DOI: 10.1039/x0xx00000x
Description of the assembled nanorods involves an analysis of the degree of orientation, represented quantitatively by the order parameter $\mathbf{S}$ as:

$$
S=\left(3 \cdot<\cos ^{2} \theta>-1\right) / 2
$$

where $\theta$ is the angle of the long axis of an individual nanorod with respect to the director $\overrightarrow{\boldsymbol{n}}$ of the ensemble. The angle brackets indicate a temporal and spatial average in the ensemble of nanorods residing in the measured volume. According to Equation 1, the order parameter $\mathbf{S}$ is 1 for a perfectly aligned system while $\mathbf{S}$ is $\mathbf{0}$ for a random ensemble. Quantitative measurement of $\mathbf{S}$ is crucial as it allows to assess the alignment process and to establish the relation between $\mathbf{S}$ and the amount of anisotropy of physical properties.

A widely used technique for analyzing assembled structures and determining $\mathbf{S}$ is to measure the induced optical birefringence $(\Delta n)$. However, in practice, determining the absolute value of $\mathbf{S}$ from $\Delta \mathrm{n}$ is a complex problem often requiring a sophisticated intrinsic- and form- birefringence modeling that relates to the structure of assembly and the intrinsic optical constants of the building blocks and 


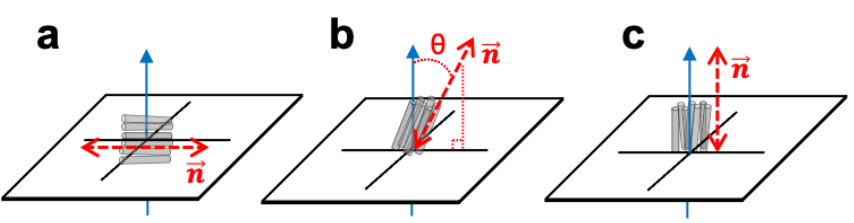

Figure 1. Schematic illustration of nanorods assembly with the alignment director (a) $\vec{n}$ parallel to the substrate (measurement plane), (b) $\vec{n}$ with out-of-plane angle $(\theta)$ and (c) $\vec{n}$ perpendicular to the substrate. Blue line indicates the direction of light propagation for measurement.

surrounding medium. ${ }^{1,26,27,28}$ Also, birefringence measurement is only useful for simple configurations where the assembly is unidirectional and $\overrightarrow{\boldsymbol{n}}$ is parallel to the measurement plane (i.e. perpendicular to the light propagation) (Figure 1a). When the assembly has an out-of-plane component of $\overrightarrow{\boldsymbol{n}}(\theta), \mathbf{S}$ cannot be deduced unless $\theta$ is known. (Figure 1b). SAXS (small-angle $x$ ray scattering) analysis also offers measurement of $\mathbf{S}$ as well as detailed information such as superlattice structure and interparticle spacing. ${ }^{25,29,30}$ However, a sophisticated scattering pattern analysis is essential to quantify $\mathbf{S}$, which is highly sensitive to the experimental and materials parameters. Moreover, SAXS instruments are heavy, costly, and not easily accessible. Measuring $\mathbf{S}$ has sometimes been made by direct observation of assembled nanorods by electron or optical microscopy. ${ }^{18,21,31,32,33}$ However, electron microscopy is destructive and cannot be made in situ in most cases, while the optical microscopy imaging is only useful when the size of particles is larger than the optical resolution.

In this study, we present a rapid, high-resolution, nondestructive, and in-situ method to measure $\mathbf{S}$, particularly the homeotropic order parameter $\left(\mathbf{S}_{\text {homeo }}\right)$ of vertically aligned nanorods assembly (Figure 1c). Homeotropic assemblies are useful in various applications. For instance, Homeotropic assemblies of semiconducting nanorods is promising for energy and sensor devices due to their improved light absorption, large surface-to-volume ratio, and increased carrier collection efficiency. ${ }^{34,35,36,37,38}$ Homeotropic assemblies of plasmonic nanorods improve the performance of surfaceenhanced Raman scattering (SERS) substrates thanks to their ability to enhance local electromagnetic fields with better uniformity and reproducibility. ${ }^{39,40}$ Measuring $\boldsymbol{S}_{\text {homeo }}$ is a key to optimize the structure and performance of such systems. Meanwhile, as $\vec{n}$ is vertical to the measurement plane, birefringence or anisotropic SAXS pattern cannot be measured with the transmitted beam.

Here, a new method utilizes the photoluminescence (PL) of trivalent europium $\left(\mathrm{Eu}^{3+}\right)$ ions doped in crystalline nanorods. ${ }^{8,9}$ Lanthanides doped in an anisotropic crystal exhibit luminescence with a distinguished polarization nature. ${ }^{41}$ As a result, an emission spectrum observed from a certain viewing angle provides information on the three-dimensional orientation of the crystal. ${ }^{9}$ When many nanocrystals are collectively assembled together, measuring the spectral degree of polarization allows to determine $\mathbf{S}$ and $\overrightarrow{\boldsymbol{n}} .^{8}$ In the present work, we demonstrate that, in the case of homeotropic assembly, $\mathbf{S}_{\text {homeo }}$ can be measured even without considering the polarization but only from the line shape of the unpolarized emission spectrum. This is a great advantage because polarization state is easily perturbed by various optical components (e.g. mirrors, filters, gratings, detector arrays, etc). The proposed method is compatible with a simple conventional microscopy setup allowing its easy implementation to various applications.

\section{Results and Discussions}

\subsection{Methodology for homeotropic order parameter analysis}

The $\mathrm{Eu}^{3+}$ luminescence manifests two different dipolar natures: electric dipole (ED) and magnetic dipole (MD) depending on each transition level. When $\mathrm{Eu}^{3+}$ ions are doped in a crystalline matrix, their main transition levels are further split by crystal-field and appear as a number of narrow sublevel peaks being considered as individual dipoles. ${ }^{41}$ As each of these sublevel peaks is polarized with a certain dipole axis, the line shape of polarized luminescence spectrum shows a drastic change when the emission is collected with a rotating analyzer. ${ }^{7,8,9}$ As a result, the $\pi$ - and $\sigma$ - polarized spectra, corresponding to the emission toward directions perpendicular to the nanorod and taken with the analyzer (polarizer in front of the detector) parallel and perpendicular to the rod axis respectively, are very different with each other for both ${ }^{5} D_{0}{ }^{7} F_{1}(M D)$ transition and ${ }^{5} D_{0}{ }^{7} F_{2}(E D)$ transition lines (Figure 2a). ${ }^{9}$ When the nanorods possess a cylindrical symmetry of their crystal structure, the $\alpha$-spectrum, corresponding to the emission along the rod axis, is isotropic (unpolarized) and its line shape is identical to the $\pi$-spectrum for ${ }^{5} D_{0}-{ }^{7} F_{1}(M D)$ transition and $\sigma$-spectrum for ${ }^{5} D_{0}{ }^{-}{ }^{7} F_{2}$ (ED) transition lines (Figure 2a). ${ }^{8,9}$ We showed previously that the orientation of either individual or ensemble of $\mathrm{Eu}^{3+}$-doped nanorods can be analyzed by polarization-resolved observation of their photoluminescence $(\mathrm{PL}){ }^{8,9}$ It was also demonstrated that the emission spectral line shape depends exclusively on the crystal structure but is independent of the morphology of nanocrystals. ${ }^{9}$ Here we demonstrate that the peculiar characteristic of $\pi-, \sigma-$, and $\alpha$ - spectra enables to determine $S_{\text {homeo of vertically aligned nanorods without using any }}$ polarization optics, which greatly simplifies the measurement and analysis.

When a single $\mathrm{Eu}^{3+}$-doped nanorod is in 3D space with arbitrary spherical angles ( $\theta$-polar and $\varphi$-azimuthal) with respect to the measurement axis (z-axis) (Figure $\mathbf{2 b}$ ), its $\mathrm{PL}$ intensities collected at two orthogonal analyzer angles $\left(I_{z x}, I_{z y}-\right.$ the two indices indicate the measurement axis and the analyzer axes respectively) are projected sums of $\pi, \sigma$, and $\alpha$ components. The $\theta$ and $\varphi$ angles can thus be calculated using the following equations. At the whole wavelength $(\lambda)$ range for both MD and ED transition lines: 
a

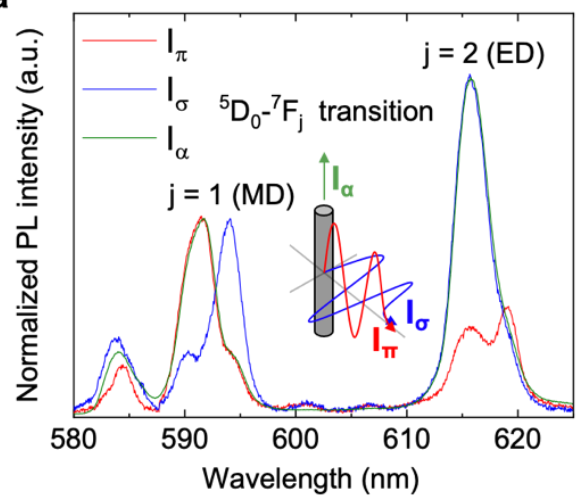

b

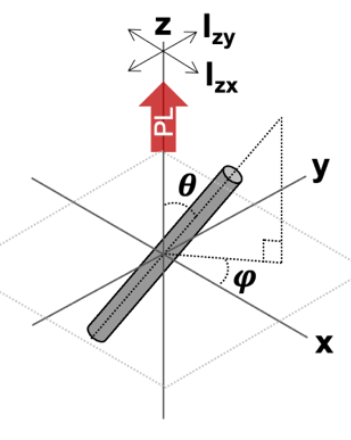

C

d

e

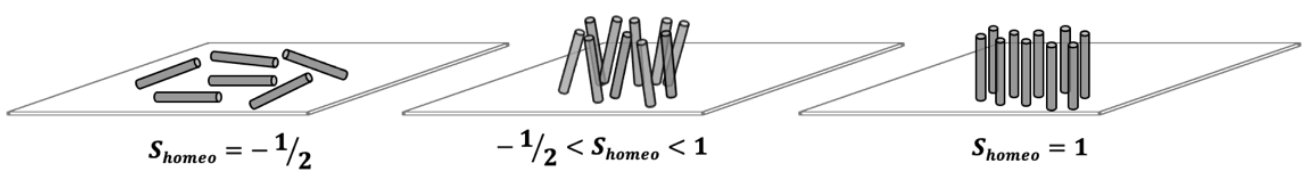

Figure 2. (a) Polarized photoluminescence (PL) spectra in $\pi, \sigma$, and $\alpha$ configurations of $\mathrm{NaYF}_{4}: \mathrm{Eu}^{3+}$ nanorods under the excitation at $394 \mathrm{~nm}\left({ }^{7} \mathrm{~F}_{0} \rightarrow{ }^{5} \mathrm{~L}_{6}\right)$. These spectra were obtained from the $\mathrm{NaYF}_{4}: \mathrm{Eu}^{3+}$ colloidal nanorods aligned under electric field. The electro-optical switching experiments are detailed in Figure S1-2 (for the $\pi$ and $\sigma$ signals) and in Section $\mathbf{2 . 2}$ (for the $\alpha$ signal). This figure is adapted from the reference. ${ }^{9}$ (b) Schematic illustration of a single nanorod in laboratory frame with an arbitrary orientation - polar and azimuthal angles $(\theta, \varphi)$ with respect to the measurement axis ( $z$-axis). The PL signal measured along the $z$-axis is a projected sum of $\pi$ and $\sigma$ signals. Schematic illustration of nanorods assembly (c) lying on the substrate (normal to the measurement axis) and (e) standing normal to the substrate (parallel to the measurement axis). (d) shows an intermediate state where the nanorods are partially oriented normal to the substrate. The value or the range of homeotropic order parameter $\mathbf{S}_{\text {homeo }}$ for each state is noted.

$I_{z y}(\lambda)=I_{\pi}(\lambda) \cdot \sin ^{2} \theta \cdot \sin ^{2} \varphi+I_{\sigma}(\lambda) \cdot \sin ^{2} \theta \cdot \cos ^{2} \varphi+$

$$
I_{\alpha}(\lambda) \cdot \cos ^{2} \theta
$$

$$
\begin{aligned}
I_{z x}(\lambda)= & I_{\pi}(\lambda) \cdot \sin ^{2} \theta \cdot \cos ^{2} \varphi+I_{\sigma}(\lambda) \cdot \sin ^{2} \theta \cdot \sin ^{2} \varphi+ \\
& I_{\alpha}(\lambda) \cdot \cos ^{2} \theta
\end{aligned}
$$

The PL collected in the measurement axis $\left(I_{z}\right)$ without analyzer is the sum of the two orthogonal polarizations $\left(I_{z x}\right.$ and $\left.I_{z y}\right)$. Therefore, Equation 2-3 can be rewritten as:

$$
\begin{aligned}
& I_{z}(\lambda)=I_{z x}(\lambda)+I_{z y}(\lambda)= \\
& \sin ^{2} \theta \cdot\left(I_{\pi}(\lambda)+I_{\sigma}(\lambda)\right)+\quad 2 \cos ^{2} \theta \cdot I_{\alpha}(\lambda)
\end{aligned}
$$

When considering an assembly of multiple nanorods, the collected PL emission is a sum of the contributions by all the nanorods in the measured focal volume. In this case, $\cos ^{2} \theta$ of a single nanorod orientation in Equation 4 can be replaced by $<\cos ^{2} \theta>$ that is a spatial and temporal mean of $\cos ^{2} \theta$ of all the nanorods residing in the focal volume during the time of measurement. Note that a normally distributed orientation $(\theta)$ can be assumed when a unidirectional electric field is competing with the thermal relaxation. In the case of homeotropic assembly (i.e. $\overrightarrow{\boldsymbol{n}}$ vertical to the substrate plane and parallel to the measurement axis, Figure 1c), measured $<\cos ^{2} \theta>$ corresponds to the distribution of the polar angles with respect to the director of alignment $\overrightarrow{\boldsymbol{n}}$. As shown in
Equation 1, the order parameter $\mathbf{S}$ is a function of $\left\langle\cos ^{2} \theta\right\rangle$. Therefore, Equation 4 can be rewritten for an assembly of nanorods using Equation 1 as:

$\frac{3}{2} I_{z}(\lambda)=\left(1-S_{\text {homeo }}\right) \cdot I_{\pi+\sigma}(\lambda)+\left(1+2 \cdot S_{\text {homeo }}\right) \cdot I_{\alpha}(\lambda)$

where $I_{\pi+\sigma}(\lambda)$ is a sum of $I_{\pi}(\lambda)$ and $I_{\sigma}(\lambda)$. In Equation 5, the PL collected with the measurement axis $\left(I_{z}\right)$ is a simple function of the homeotropic order parameter $\left(\mathbf{S}_{\text {homeo }}\right)$ and the reference emission intensities $\left(I_{\pi}, I_{\sigma}\right.$ and $\left.I_{\alpha}\right)$. Hence, once $I_{\pi+\sigma}(\lambda)$ and $I_{\alpha}(\lambda)$ - the intrinsic reference spectra of the given crystal structure of nanorods - are known, $\mathbf{S}_{\text {homeo }}$ can be obtained by a simple measurement of the unpolarized emission spectrum $\left(I_{z}(\lambda)\right)$.

The value of $\mathbf{S}_{\text {homeo }}$ is 1 for a perfect homeotropic alignment (Figure 2e) and 0 for a random orientation. The minimum of $S_{\text {homeo }}$ is $-1 / 2$ when all the nanorods are lying on the substrate plane normal to the measurement axis (Figure 2c). In this case, $<\cos ^{2} \theta>$ is zero and thus $I_{z}$ is equal to $I_{\pi+\sigma}$ without contribution of $I_{\alpha}$. So, the reference spectrum, $I_{\pi+\sigma}(\lambda)$, can easily be collected without using an analyzer from a sample of nanorods lying on a substrate. By a separate experiment observing electrically in-plane-aligned nanorods with an analyzer (Figure S1-2), it was confirmed that the sum of the two orthogonally polarized $\pi$ - and $\sigma$-spectra $\left(I_{\pi}(\lambda)+I_{\sigma}(\lambda)\right)$ is identical to the $\mathrm{I}_{\pi+\sigma}(\lambda)$ spectrum of randomly deposited nanorods (Figure $\mathbf{S 3}$ ). The other reference spectrum, $I_{\alpha}(\lambda)$, can be obtained also without analyzer from a sample with a perfect vertical alignment as $I_{z}=2 I_{\alpha}$ for $S_{\text {homeo }}=1$ (Figure 2e). As the experimental spectrum $\left(I_{z}(\lambda)\right)$ and the two reference spectra 


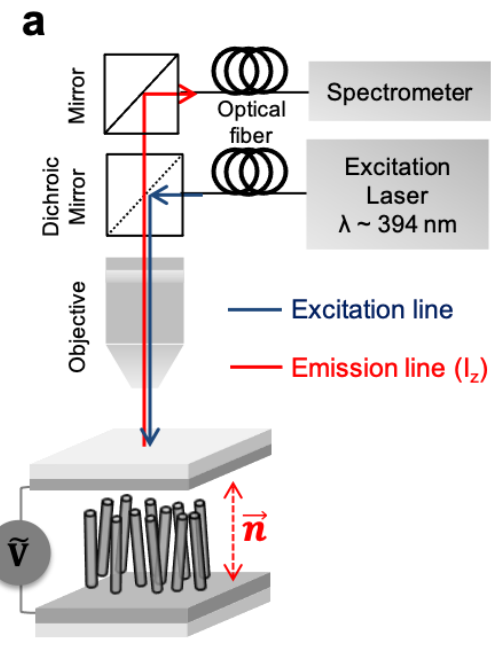

b

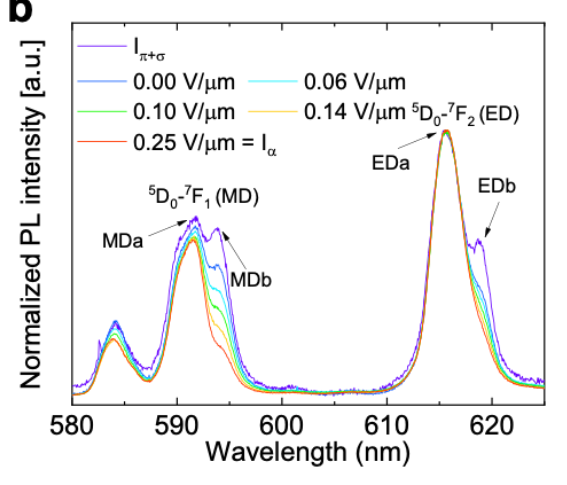

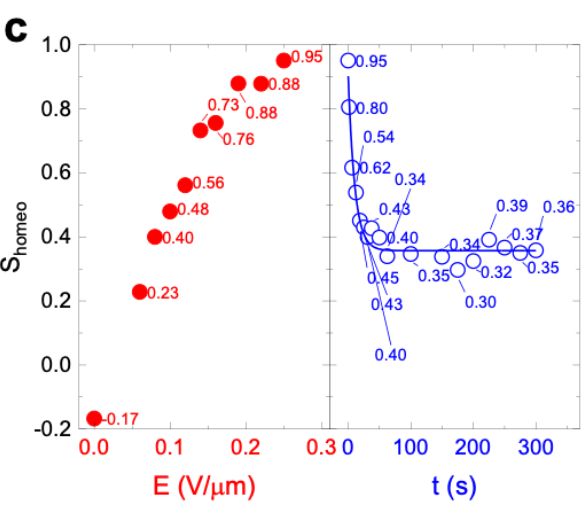

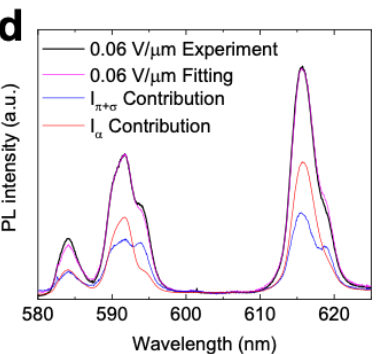

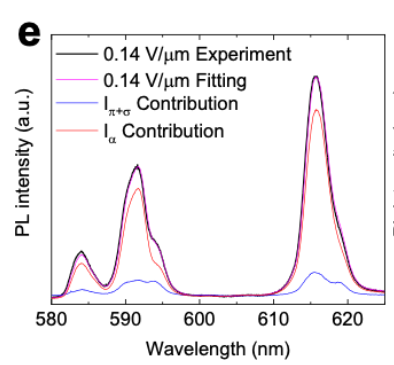

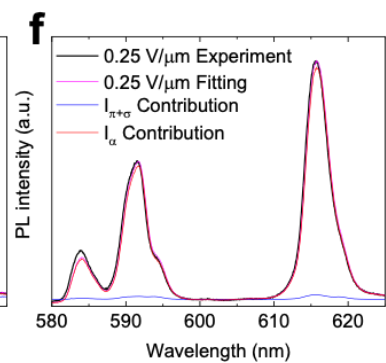

Figure 3. (a) A scheme of the conventional fluorescence microscopy setup used to conduct the order parameter $\mathbf{S}_{\text {homeo }}$ analysis of the homeotropic NaYF $\mathrm{F}_{4}$ Eu nanorods assembly under electric-field (E-field). A fiber-coupled diode laser at $394 \mathrm{~nm}$ (corresponding to the ${ }^{7} \mathrm{~F}_{0} \rightarrow{ }^{5} \mathrm{~L}_{6}$ transition of Eu ${ }^{3+}$ ) was used as an excitation source. The PL emission $\left({ }^{5} \mathrm{D}_{0} \rightarrow{ }^{7} \mathrm{~F}_{\mathrm{J}}\right.$ transitions) was observed also with a fiber-coupled spectrometer. (b) The collected PL emission spectra with gradually increased E-field strength $(E)$. $I_{\pi+\sigma}$ indicates a reference spectrum taken with randomly deposited nanorods lying on the substrate. All the spectra were collected without polarizer and normalized to the peak with highest intensity at $\lambda \sim 615 \mathrm{~nm}$. (c) A plot of the calculated homeotropic order parameter $\mathbf{S}_{\text {homeo }}$ as a function of $E$ (red, left panel) and of the relaxation time ( $t$ ) after saturation at $E=0.25 \mathrm{~V} / \mu \mathrm{m}$ and turning off the $E$-field (blue, right panel). The spectral fitting of the emission spectra for different E-field intensities of (d) 0.06 , (e) 0.14 and (f) $0.25 \mathrm{~V} / \mu \mathrm{m}$ with the reference spectra $\left(I_{\pi+\sigma}\right.$ and $\left.I_{\alpha}\right)$ providing the value of $\boldsymbol{S}_{\text {homeo }}$ according to the Equation $\mathbf{5}$.

$\left(I_{\pi+\sigma}(\lambda)\right.$ and $\left.I_{\alpha}(\lambda)\right)$ can be obtained without analyzer, this method does not require consideration of polarization during the experiment nevertheless it bases on the intrinsic anisotropy of the luminescence of $\mathrm{Eu}^{3+}$-doped nanorods. This allows a facile and robust analysis of $\boldsymbol{S}_{\text {homeo }}$ eliminating the measurement errors and complexity due to the polarizing optics. It is also worth noting that this methodology is free from consideration of the structural factors other than $\boldsymbol{S}_{\text {homeo }}$ such as volume fraction of the nanorods, sample thickness, and other intrinsic material properties. The geometrical analysis of $\boldsymbol{S}_{\text {homeo }}$ can thus be made through a purely spectroscopic method.

\subsection{In-situ analysis of $S_{\text {homeo }}$ in electric field-induced nanorod assembly}

As a proof of concept device, we prepared a homeotropic electro-optical cell in which a colloidal suspension of $\mathrm{Eu}^{3+}$ doped monocrystalline sodium yttrium fluoride $\left(\mathrm{NaYF}_{4}: \mathrm{Eu}\right)$ nanorods is inserted. An AC (alternating current) electric field (E-field) applied between the top and bottom substrate could induce the vertically oriented nanorods assembly, which could be reversibly controlled by adjusting the intensity of E-field. $\mathrm{NaYF}_{4}$ was selected as it is known as an efficient host crystal for doping lanthanide ions. ${ }^{42,43} \mathrm{~A}$ micrometer-long $\mathrm{NaYF}_{4}: \mathrm{Eu}$ nanorods were synthesized by a wet-chemical method ${ }^{44}$, surface-functionalized with citrate molecules ${ }^{45}$, and stably dispersed in ethylene glycol. The degree of orientation of the nanorods under the applied E-field changed depending on the E-field strength. The PL signal was collected simultaneously with the modulation of the applied E-field in order to quantitatively determine $\boldsymbol{S}_{\text {homeo }}$ at each moment. The simple fluorescence microscopy setup used is schematized in Figure 3a.

The method requires a preset of reference spectra $\left(I_{\pi+\sigma}(\lambda)\right.$ and $I_{\alpha}$ ), as discussed above. Firstly, $\mathrm{NaYF}_{4}$ :Eu nanorods were spin coated on the glass substrate and heated until the solvent was evaporated. The nanorods deposited in this way were perfectly lying on the glass substrate, which was used to collect $I_{\pi+\sigma}(\lambda)$ spectrum (Figure $3 \mathbf{b}$ ). Subsequently, the $I_{\alpha}$ signal was collected from perfectly vertically aligned nanorods assembly under a strong E-field $(E>0.25 \mathrm{~V} / \mu \mathrm{m})$ saturating the increase of $\mathbf{S}_{\text {homeo }}$ (Figure $\mathbf{3 b}$ ). The $\mathrm{PL}$ spectra collected under varied E-field strength between $0.00 \mathrm{~V} / \mu \mathrm{m}$ and $0.25 \mathrm{~V} / \mu \mathrm{m}$ show a clear line shape change (Figure $\mathbf{3 b}$ ). As the E-field strength increases, in the ${ }^{5} \mathrm{D}_{0}-{ }^{7} \mathrm{~F}_{1}$ (MD) transition range, the relative intensity of the $M D_{b}$ peak at $593.7 \mathrm{~nm}$ decreases compared to the almost constant intensity of the $\mathrm{MD}_{\mathrm{a}}$ peak at $591.4 \mathrm{~nm}$. While in the ${ }^{5} \mathrm{D}_{0}-{ }^{7} \mathrm{~F}_{2}$ (ED) transition range, the relative intensity of the $\mathrm{ED}_{\mathrm{b}}$ peak at $619.0 \mathrm{~nm}$ decreases compared to the constant intensity of the $E D_{a}$ peak at 615.3 $\mathrm{nm}$. When the E-field strength was increased higher than 0.25 
$\mathrm{V} / \mu \mathrm{m}$, no longer spectral change was observed. The PL spectrum observed at this saturation level was identical to the $\pi$-spectrum in the ${ }^{5} \mathrm{D}_{0^{-}}{ }^{7} \mathrm{~F}_{1}(\mathrm{MD})$ transition range and to the $\sigma$ spectrum in the ${ }^{5} D_{0}-{ }^{7} F_{2}$ (ED) transition range (Figure 2a), which is thus equal to the $\alpha$-spectrum ${ }^{8,9}$ and confirms that $\mathbf{S}_{\text {homeo }}$ is close to 1 at this state. Note that, compared to the typical organic liquid crystals used for electro-optical devices, 0.25 $\mathrm{V} / \mu \mathrm{m}$ is about one order of magnitude lower E-field strength required to fully control their orientations. Such an efficient control with a moderate E-field may be useful for further device application using colloidal nanorod systems.

By fitting the reference spectra $\left(I_{\pi+\sigma}\right.$ and $\left.I_{\alpha}\right)$ on the experimental spectra using Equation $\mathbf{5}$, the $\boldsymbol{S}_{\text {homeo }}$ values were calculated and plotted as a function of E-field strength (Figure 3c-left panel). Initially, when $E=0 \mathrm{~V} / \mu \mathrm{m}$ where the dispersed nanorods exhibit a random Brownian motion without an alignment effect by the electric field, the calculated $\mathbf{S}_{\text {homeo }}$ was -0.17 , which is lower than 0 for a perfectly random orientation. This seems to be due to the in-plane capillary force that induces partial orientation of the nanorods parallel to the substrate plane when injecting them into the thin gap ( 20 $\mu \mathrm{m}$ ) of the electro-optical cell. The $\boldsymbol{S}_{\text {homeo }}$ value gradually increases as a function of the E-field strength. Figure 3d-f are examples of the fitting analysis showing that the contribution of $I_{\alpha}$ (red lines) compared to $I_{\pi+\sigma}$ (blue lines) increases when increasing the E-field strength. When $E=0.25 \mathrm{~V} / \mu \mathrm{m}$, the contribution of $I_{\pi+\sigma}$ is almost negligible and $I_{\alpha}$ occupies most of the emission spectrum providing $\mathbf{S}_{\text {homeo }}=0.95$ (Figure 3f).

The PL spectrum was continuously observed after turning off the E-field in order to examine the relaxation behavior of the aligned nanorods. Without E-field, nanorods are subject to a randomization of their orientation due to the Brownian motion. The calculated $\mathbf{S}_{\text {homeo }}$ values as a function of time after turning off the E-field are shown in Figure 3c-right panel. The corresponding PL spectra are shown in Figure S4. These experimental points fit with an exponential decay curve $\left(y=A \cdot \exp \left(-x / t_{1}\right)+y_{0}\right)$ with a relatively long decay time $(\tau=7.4 \mathrm{~s})$. We attribute this slow relaxation dynamics to the relatively large size of the nanorods (Length $=1184 \pm 430 \mathrm{~nm}$, Diameter $=117 \pm 24 \mathrm{~nm})$ and the viscosity of ethylene glycol $\left(1.61 \cdot 10^{-2}\right.$ $\mathrm{Pa} \cdot \mathrm{s})$ as a dispersion medium. Theoretical rotational diffusion coefficient is given as:

$$
\Omega=\frac{3 k_{b} T}{16 \pi \eta_{0} a^{3}}\left(-1+2 \ln \frac{2 a}{b}\right)
$$

where $k_{b}$ is Boltzmann constant, $\eta_{0}$ is solvent viscosity, $a$ and $b$ are respectively the half-length and equatorial radius of the ellipsoidal particle. ${ }^{46}$ The $\Omega$ calculated for our $\mathrm{NaYF}_{4}: \mathrm{Eu}$ nanorods dispersed in ethylene glycol is $0.37 \mathrm{~s}^{-1}$. It is in good accordance with the experimentally obtained relaxation time of $7.4 \mathrm{~s}\left(\cong 0.14 \mathrm{~s}^{-1}\right)$ regarding that $\Omega$ is highly sensitive to the particle size while our nanorods have a certain polydispersity. Interestingly, the relaxation of $\mathbf{S}_{\text {homeo }}$ showed a saturation at around 0.35 , meaning that the orientation of nanorods were

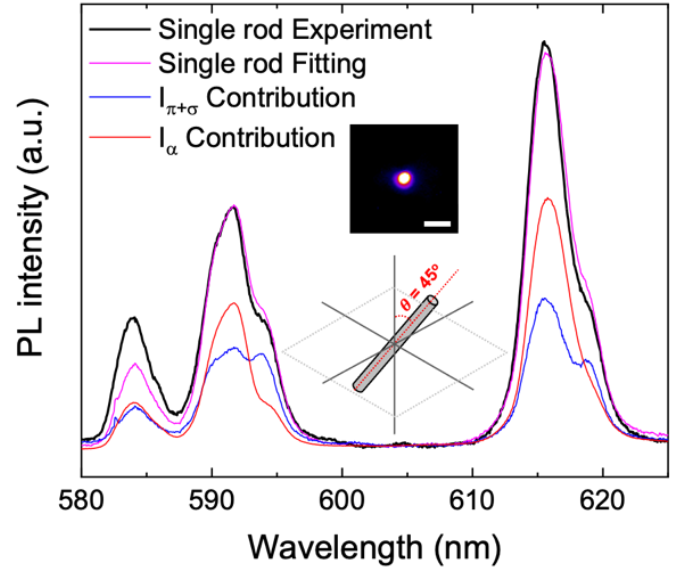

Figure 4. The emission spectrum of a single $\mathrm{NaYF}_{4}$ :Eu nanorod embedded in a polymer film with an arbitrary orientation (black line) and its fitting analysis with the reference spectra determining the polar angle $\theta$ of a single nanorod according to Equation 4. The inset picture is a photograph of the PL emission of the single nanorod captured with a CCD camera (scale bar: $2 \mu \mathrm{m}$ ). The inset scheme illustrates a single nanorod in a laboratory frame with a determined polar angle $\theta$ from the fitting analysis.

not completely randomized and there was a memory effect maintaining partially the orientation along the previously applied E-field. It is presumably due to some portion of nanorods that were dragged by the E-field and vertically attached on the electrode surface losing their mobility. Further study is required to explain and control this phenomenon. It is also worth noting that there exists fluctuation of $\boldsymbol{S}_{\text {homeo }}$ from 0.30 to 0.39 when measured at different moments even after the saturation without E-field. There seems to be thus a continuous thermal agitation and the presented method is quite sensitive to these small changes (see Figure S4b).

The accuracy of the calculation of $\mathbf{S}_{\text {homeo }}$ according to Equation $\mathbf{5}$ can be evaluated from its simulated profile as a function of relative contributions of $I_{\pi_{+\sigma}}$ and $I_{\alpha}$ (Figure S5). One can expect that the accuracy would be less good at the extremities where $\mathbf{S}_{\text {homeo }}$ is near 1 or $-1 / 2$. This implies that there might be some error on the calculated value of $\mathbf{S}_{\text {homeo }}=0.95$ near saturation $(E$ $=0.25 \mathrm{~V} / \mu \mathrm{m})$. In order to minimize errors, stable and good spectral resolution and correct baseline treatment of the measured spectra are necessary.

\subsection{Analysing $\theta$ of a single nanorod}

The same method can be applied to measure the out-of-plane orientation $(\theta)$ of a single $\mathrm{NaYF}_{4}$ :Eu nanorod (Figure $2 \mathbf{2 b}$ ). A randomly oriented single nanorod was immobilized in a $\sim 8 \mu \mathrm{m}$ thick PVA (polyvinyl alcohol) polymer film. The PL image of a single nanorod captured by a CCD camera (Figure 4, inset picture) shows an almost circular shape due to the nanorod size smaller than the spatial resolution of the camera. So, any information about the nanorod orientation cannot be retrieved from the image. However, the line shape of the emission spectrum from the same nanorod enables calculating a polar angle $(\theta)$ of the nanorod orientation with respect to the 
measurement axis according to Equation 4 (Figure 4, inset scheme). The calculated value of $\theta$ is $45^{\circ}$ as noted in the figure. Calculating azimuthal angle $\varphi$ from the line shape of photoluminescence is also possible if the polarization of the emission spectrum is measured. The original methods to measure the 3D orientation of single nanorods employing polarizing optics is presented in our previous work. ${ }^{9}$

\section{Conclusions}

We have demonstrated that the homeotropic order parameter ( $\left.\mathbf{S}_{\text {homeo }}\right)$ of vertically aligned nanorods assembly can be measured by a spectroscopic analysis of the photoluminescence emission of doped europium ions. The presented method is based on the optical anisotropy inherent in lanthanide luminescence resulting in the variation of the emission spectral line shape as a function of $\boldsymbol{S}_{\text {homeo. }}$. It was shown that a precise measurement of $\boldsymbol{S}_{\text {homeo }}$ can be performed using a very simple microscope configuration without using any polarizing optical component. We have used $\mathrm{NaYF}_{4}: \mathrm{Eu}$ nanorods as a proof of concept material but any other materials doped with lanthanides exhibiting both MD and ED transitions can be used to perform the proposed orientation analysis. ${ }^{9}$ Varying degree of orientation in nanorods assembly under externally applied electric field was investigated in real time. The demonstrated results confirm that a facile and costeffective but quantitative analysis of $\boldsymbol{S}_{\text {homeo }}$ can be made. A crucial advantage of this method is that the measurement domain can be as small as the focal volume of optical microscopy $\left(<0.1 \mu^{3}\right)$, which has never been achieved by other techniques to assess the directional assembly of nanoobjects. Furthermore, a rapid in-situ analysis can be realized in a non-destructive way by this novel spectroscopic method. It is thus promising for investigations of a variety of collective dynamics of nanoscale materials (e.g. self-assembly, directed assembly, liquid crystalline behavior, thermal motions, vertical anisotropic growth, etc) with a closer look into their micro- and nano-scale domains.

\section{Experimental Section}

\subsection{Nanorod synthesis and sample preparation}

$\mathrm{NaYF}_{4}$ :Eu nanorods, with $5 \%$ of Eu doping were solvothermally synthesized following the procedure reported in the reference. ${ }^{44}$ Briefly, $45 \mathrm{mmol}(1.8 \mathrm{~g})$ of $\mathrm{NaOH}$ is dissolved in $6 \mathrm{~mL}$ of water and mixed with $15 \mathrm{~mL}$ of ethanol (EtOH) and $30 \mathrm{~mL}$ of oleic acid (OA) under vigorous stirring. To the resulting mixture, $0.95 \mathrm{mmol}(288 \mathrm{mg})$ of $\mathrm{Y}(\mathrm{Cl})_{3}, 6 \mathrm{H}_{2} \mathrm{O}, 0.05 \mathrm{mmol}(18 \mathrm{mg})$ of $\mathrm{Eu}(\mathrm{Cl})_{3} 6 \mathrm{H}_{2} \mathrm{O}$ and $10.2 \mathrm{mmol}\left(377 \mathrm{mg}\right.$ ) of $\mathrm{NH}_{4} \mathrm{~F}$ dissolved in $4 \mathrm{~mL}$ of water were added. The solution was then heated in an autoclave at $200^{\circ} \mathrm{C}$ for 24 hours under stirring. The obtained nanoparticles were washed with water and $\mathrm{EtOH}$ several times and recovered as a white powder after drying in vacuum. For optical experiments, the nanoparticles were further sonicated in $2 \mathrm{~mL}$ of aqueous citrate solution $(0.2 \mathrm{M})$ and centrifuged several times to fully exchange the surface-ligand (OA) with citrate ions. ${ }^{45}$ The washed nanoparticles with $\mathrm{EtOH}$ were well dispersed in water or ethylene glycol (EG). As-obtained $\mathrm{NaYF}_{4}: \mathrm{Eu}$ nanorods are hexagonal phase monocrystals and polydisperse with the size of $1184 \pm 430 \mathrm{~nm}$ in length (L) and $117 \pm 24 \mathrm{~nm}$ in diameter (D) and with an average aspect ratio (L/D) of $\sim 10$. All the electro-optical experiments were performed using $\mathrm{NaYF}_{4}$ :Eu nanorods dispersed in EG. The single particle sample embedded in the polymer film was prepared by drop-casting an aqueous solution of polyvinyl alcohol (MW 22000) $\left(0.1 \mathrm{~g} / \mathrm{mL}\right.$ ) and $\mathrm{NaYF}_{4}:$ Eu nanorods together on a glass substrate followed by vacuum drying. Scanning electron microscopy (SEM) picture and X-ray diffraction (XRD) pattern of $\mathrm{NaYF}_{4}$ :Eu nanorods used in this study are presented in Figure $\mathbf{S 6}$ and also in our previous study. ${ }^{9}$

\subsection{Electro-Optical switching}

For the measurement of the PL spectra in the $\pi, \sigma$, and $\alpha$ configurations, nanorods were aligned using electro-optical (E/O) cells. For the in-plane alignment ( $\pi$ and $\sigma$ configurations) (Figure S1,2), an in-plane switching cell was made with gold thin film electrodes deposited with a parallel gap (width $\sim 150$ $\mu \mathrm{m})$ and a $60 \mu \mathrm{m}$-thick spacer. $20 \mu \mathrm{L}$ of nanorods dispersion in ethylene glycol was dropped at the center and covered with a glass coverslip. An AC electric field $(1 \mathrm{kHz}, 0.47 \mathrm{~V} / \mu \mathrm{m})$ was applied to fully align the nanorods between the gap. For the homeotropic alignment ( $\alpha$ configuration), a commercial homeotropic E/O cell (Instec, Inc.) was used also with an AC electric field ( $500 \mathrm{kHz}, 0.25 \mathrm{~V} / \mu \mathrm{m})$ (Figure 3a).

\subsection{Optical spectroscopy}

The photoluminescence (PL) spectra of $\mathrm{NaYF}_{4}: \mathrm{Eu}$ in $\mathrm{E} / \mathrm{O}$ cell were obtained using a upright optical microscope (Olympus $B X$ $51 \mathrm{WI}$, Objective: magnification $=20 x, N A=0.45$ ) (Figure 2,3, Figure S2b-f, Figure S3,4). A fiber-coupled monochromatic diode laser (Oxxius, $\lambda_{\text {center }}=394 \mathrm{~nm}, \mathrm{FWHM}=0.7 \mathrm{~nm}$ ) was used as an excitation source matching with the $7 \mathrm{~F}^{\circ} \rightarrow 5 \mathrm{~L}^{6}$ transition of $\mathrm{Eu}^{3+}$. The PL signals were collected with a fibercoupled spectrometer (SpectraPro-300i, Princeton Instrument equipped with LN/CCD-1100-PP camera). The PL spectra of single $\mathrm{NaYF}_{4}: \mathrm{Eu}$ nanorods were collected using the same optical setup with higher magnification (magnification $=100 \mathrm{x}$, $N A=0.9)$ (Figure S2f, Figure 4). The microscopy setup is schematized in Figure 3a. The baselines of all the photoluminescence spectra were taken as a flat line with averaged intensity between $600 \mathrm{~nm}$ and $608 \mathrm{~nm}$ where $\mathrm{Eu}^{3+}$ luminescence is not present.

\section{Author Contributions}

J-M.K., T.G. and J-W.K. designed the study. J-M.K. and K.L. synthesized and characterized the nanoparticles. J-M.K. and JW.K. prepared the optical set-ups. J-M.K. performed the electro-switching experiment and fluorescence microscopy. JM.K. analysed the data. T.G. and J-W.K. supervised the 
research. The manuscript was written through contributions of all authors.

\section{Conflicts of interest}

There are no conflicts to declare.

\section{Acknowledgements}

This research was supported by the French national research agency (ANR) in the framework of the SpecTra and Kineharvest project (ANR-16-CE24-0014-01, ANR- 19-CE09-0033), and by Fondation Recherche Médicale (DCM20181039556, programme Chimie pour la Médecine).

\section{References}

${ }^{1}$ J. Kim, L. Martinelli, K. Lahlil, J.-P. Boilot, T. Gacoin and J. Peretti, Applied Physics Letters, 2014, 105, 061102.

2 J. Kim, J. Peretti, K. Lahlil, J.-P. Boilot and T. Gacoin, Advanced Materials, 2013, 25, 3295-3300.

${ }^{3}$ A. N. Morozovska, E. A. Eliseev and M. D. Glinchuk, Physical Review B, 2006, 73, 214106.

${ }^{4}$ K. Batra, N. Sinha, S. Goel, H. Yadav, A. J. Joseph and B. Kumar, Journal of Alloys and Compounds, 2018, 767, 1003-1011.

5 J.-J. Wu, S.-C. Liu and M.-H. Yang, Applied Physics Letters, 2004, 85, 1027-1029.

${ }^{6}$ K. Ip, R. M. Frazier, Y. W. Heo, D. P. Norton, C. R. Abernathy, S. J. Pearton, J. Kelly, R. Rairigh, A. F. Hebard, J. M. Zavada and R. G. Wilson, Journal of Vacuum Science \& Technology B: Microelectronics and Nanometer Structures Processing, Measurement, and Phenomena, 2003, 21, 1476-1481.

${ }^{7}$ E. Chaudan, J. Kim, S. Tusseau-Nenez, P. Goldner, O. L. Malta, J. Peretti and T. Gacoin, Journal of the American Chemical Society, 2018, 140, 9512-9517.

${ }^{8}$ J. Kim, S. Michelin, M. Hilbers, L. Martinelli, E. Chaudan, G. Amselem, E. Fradet, J.-P. Boilot, A. M. Brouwer, C. N. Baroud, J. Peretti and T. Gacoin, Nature Nanotechnology, 2017, 12, 914.

9 J. Kim, R. Chacón, Z. Wang, E. Larquet, K. Lahlil, A. Leray, G. Colasdes-Francs, J. Kim and T. Gacoin, Nature Communications, 2021, In Press.

${ }^{10} \mathrm{~S}$. A. Maier, Plasmonics: fundamentals and applications, Springer Science \& Business Media, 2007.

${ }^{11}$ S. Park, H. Mundoor, B. Fleury, P. Davidson, J. van de Lagemaat and I. I. Smalyukh, Advanced Optical Materials, 2019, 7, 1900041.

${ }^{12}$ Y. Xie, Y. Li, G. Wei, Q. Liu, H. Mundoor, Z. Chen and I. I. Smalyukh, Nanoscale, 2018, 10, 4218-4227.

${ }^{13}$ J. Kim, A. de la Cotte, R. Deloncle, S. Archambeau, C. Biver, J.-P. Cano, K. Lahlil, J.-P. Boilot, E. Grelet and T. Gacoin, Advanced Functional Materials, 2012, 22, 4949-4956.

${ }^{14}$ J. L. Baker, A. Widmer-Cooper, M. F. Toney, P. L. Geissler and A. P. Alivisatos, Nano Letters, 2010, 10, 195-201.

${ }^{15}$ M. Zanella, R. Gomes, M. Povia, C. Giannini, Y. Zhang, A. Riskin, M. Van Bael, Z. Hens and L. Manna, Advanced Materials, 2011, 23, 2205-2209.

${ }^{16}$ S. Ahmed and K. M. Ryan, Nano Letters, 2007, 7, 2480-2485.

${ }^{17}$ C. Hamon, M. Postic, E. Mazari, T. Bizien, C. Dupuis, P. EvenHernandez, A. Jimenez, L. Courbin, C. Gosse, F. Artzner and V. Marchi-Artzner, ACS Nano, 2012, 6, 4137-4146.

${ }^{18}$ H. Hu, M. Pauly, O. Felix and G. Decher, Nanoscale, 2017, 9, 13071314.

${ }^{19}$ T. Börzsönyi, B. Szabó, G. Törös, S. Wegner, J. Török, E. Somfai, T. Bien and R. Stannarius, Physical Review Letters, 2012, 108, 228302.

${ }^{20}$ K. M. Ryan, A. Mastroianni, K. A. Stancil, H. Liu and A. P. Alivisatos, Nano Letters, 2006, 6, 1479-1482.

${ }^{21}$ Z. Hu, M. D. Fischbein, C. Querner and M. Drndić, Nano Letters, 2006, 6, 2585-2591.

${ }^{22}$ A. de la Cotte, P. Merzeau, J. W. Kim, K. Lahlil, J.-P. Boilot, T. Gacoin and E. Grelet, Soft Matter, 2015, 11, 6595-6603.

${ }^{23}$ B. J. Lemaire, P. Davidson, J. Ferré, J. P. Jamet, P. Panine, I. Dozov and J. P. Jolivet, Physical Review Letters, 2002, 88, 125507.

${ }^{24}$ M. Wang, C. Gao, L. He, Q. Lu, J. Zhang, C. Tang, S. Zorba and Y. Yin, Journal of the American Chemical Society, 2013, 135, 1530215305.

${ }^{25}$ D. van der Beek, A. V. Petukhov, P. Davidson, J. Ferré, J. P. Jamet, H. H. Wensink, G. J. Vroege, W. Bras and H. N. W. Lekkerkerker, Physical Review E, 2006, 73, 041402

${ }^{26}$ V. Twersky, Journal of the Optical Society of America, 1975, 65, 239-245.

${ }^{27}$ R. Oldenbourg and T. Ruiz, Biophysical journal, 1989, 56, 195-205.

${ }^{28}$ Bragg, W. L.; Pippard, A. B., The form birefringence of macromolecules. Acta Crystallographica, 1953, 6, 865-867.

${ }^{29}$ J. Connolly, J. S. v. Duijneveldt, S. Klein, C. Pizzey and R. M. Richardson, Journal of Physics: Condensed Matter, 2007, 19, 156103.

${ }^{30}$ K.-i. Murata and H. Tanaka, Proceedings of the National Academy of Sciences, 2015, 112, 5956-5961.

${ }^{31}$ W. Ahmed, E. S. Kooij, A. van Silfhout and B. Poelsema, Nano Letters, 2009, 9, 3786-3794.

${ }^{32}$ R. Baiyasi, M. J. Gallagher, L. A. McCarthy, E. K. Searles, Q. Zhang, S. Link and C. F. Landes, The Journal of Physical Chemistry A, 2020, 124, 5262-5270.

${ }^{33}$ A. Mohraz and M. J. Solomon, Langmuir, 2005, 21, 5298-5306. 
${ }^{34}$ Z. Fan, H. Razavi, J.-w. Do, A. Moriwaki, O. Ergen, Y.-L. Chueh, P. W. Leu, J. C. Ho, T. Takahashi, L. A. Reichertz, S. Neale, K. Yu, M. Wu, J. W. Ager and A. Javey, Nature Materials, 2009, 8, 648-653.

${ }^{35}$ M. Law, L. E. Greene, J. C. Johnson, R. Saykally and P. Yang, Nature Materials, 2005, 4, 455-459.

${ }^{36}$ O. K. Varghese, M. Paulose and C. A. Grimes, Nature Nanotechnology, 2009, 4, 592-597.

${ }^{37}$ Y. Li, T. Takata, D. Cha, K. Takanabe, T. Minegishi, J. Kubota and K. Domen, Advanced Materials, 2013, 25, 125-131.

38 J. X. Wang, X. W. Sun, Y. Yang, H. Huang, Y. C. Lee, O. K. Tan and L. Vayssieres, Nanotechnology, 2006, 17, 4995-4998.

${ }^{39}$ B. Peng, G. Li, D. Li, S. Dodson, Q. Zhang, J. Zhang, Y. H. Lee, H. V. Demir, X. Yi Ling and Q. Xiong, ACS Nano, 2013, 7, 5993-6000.

${ }^{40}$ Q. Liao, C. Mu, D.-S. Xu, X.-C. Ai, J.-N. Yao and J.-P. Zhang, Langmuir, 2009, 25, 4708-4714.

${ }^{41}$ K. Binnemans, Coordination Chemistry Reviews, 2015, 295, 1-45.

${ }^{42}$ R. Kumar, M. Nyk, T. Y. Ohulchanskyy, C. A. Flask and P. N. Prasad, Advanced Functional Materials, 2009, 19, 853-859.

${ }^{43}$ K. W. Krämer, D. Biner, G. Frei, H. U. Güdel, M. P. Hehlen and S. R. Lüthi, Chemistry of Materials, 2004, 16, 1244-1251.

${ }^{44}$ X. Wang, J. Zhuang, Q. Peng and Y. Li, Nature, 2005, 437, 121-124.

${ }^{45}$ T. Cao, T. Yang, Y. Gao, Y. Yang, H. Hu and F. Li, Inorganic Chemistry Communications, 2010, 13, 392-394.

${ }^{46}$ R. Cerf and H. A. Scheraga, Chemical Reviews, 1952, 51, 185-261. 